\title{
Inhibition of microRNA-155 ameliorates cardiac fibrosis in the process of angiotensin II-induced cardiac remodeling
}

\author{
YUZHEN WEI ${ }^{*}$, XIAOFEI YAN ${ }^{*}$, LIANHUA YAN, FEN HU, WENHAN MA, \\ YA WANG, SHUAI LU, QIUTANG ZENG and ZHAOHUI WANG \\ Laboratory of Cardiovascular Immunology, Institute of Cardiology, Union Hospital, Tongji Medical \\ College, Huazhong University of Science and Technology, Wuhan, Hubei 430022, P.R. China
}

Received October 8, 2016; Accepted July 20, 2017

DOI: $10.3892 / \mathrm{mmr} .2017 .7584$

\begin{abstract}
Cardiac fibrosis triggered by pressure overload represents one of the major challenges in the treatment of cardiovascular diseases. MicroRNA (miRNA/miR)-155, a member of the small RNA family, has previously been demonstrated to be associated with cardiac inflammation. However, the effect of miR-155 on cardiac fibrosis induced by angiotensin II (Ang II), particularly in cardiac fibroblasts, requires further investigation. The present study aimed to investigate the effect of miR-155 in Ang II-induced cardiac fibrosis using animal models and cardiac fibroblasts. Animal models were established in male miR-155/- and wild-type (WT) $\mathrm{C} 57 \mathrm{Bl} / 6 \mathrm{~J}$ mice (10-12 weeks old) by Ang II infusion using subcutaneously implanted minipumps. After 8 weeks of Ang II infusion, the results demonstrated that the deletion of miR-155 in mice markedly ameliorated ventricular remodeling compared with WT mice, as demonstrated by restricted inflammatory responses, decreased heart size, improved cardiac function and reduced myocardial fibrosis. In vitro, overexpression of miR-155 in cardiac fibroblasts led to significantly increased fibroblast to myofibroblast transformation. However, this effect was abrogated by miR-155 silencing. In conclusion, the results of the present study indicate that genetic loss of miR-155 in mice ameliorates cardiac fibrotic remodeling following pressure overload. Therefore, inhibiting miR-155 may have potential as an adjunct to reduce cardiac inflammation in the treatment of cardiac fibrosis.
\end{abstract}

Correspondence to: Professor Zhaohui Wang, Laboratory of Cardiovascular Immunology, Institute of Cardiology, Union Hospital, Tongji Medical College, Huazhong University of Science and Technology, 1277 Jiefang Avenue, Wuhan, Hubei 430022, P.R. China

E-mail: wuxiaohongtian@163.com

*Contributed equally

Key words: microRNA-155, cardiac remodeling, suppressor of cytokine signaling 1 , cardiac fibrosis

\section{Introduction}

Cardiac fibrosis, which may be induced by various factors in cardiac tissue, is a result of excessive deposition of extracellular matrix (ECM), and eventually leads to the destruction of the physiological cardiac tissue architecture and heart failure (1). Furthermore, as the fibrous tissue increases, myocardial stiffness increases, which may theoretically lead to decreased myocardial shortening. Systolic and diastolic cardiac functions are associated with the degree of cardiac fibrosis $(2,3)$. Fibroblasts are the primary producers of ECM and contribute substantially to myocardial fibrosis (4). During cardiac remodeling, fibroblasts differentiate into myofibroblasts, which are the primary cell type involved in the reorganization of the ECM. Myofibroblasts exhibit important contractile and secretory functions, and are characterized by rapid proliferation and are $\alpha$-smooth muscle actin ( $\alpha$-SMA)-positive (5). Various growth factors may participate in this process, including angiogenic factors, proteolytic enzymes and fibrogenic cytokines (6). As one of the major mediators of cardiac inflammation, it has been demonstrated that Angiotensin II (Ang II) regulates vascular constriction and influence cardiac function, particularly in the process of cardiac remodeling (1,7).

MicroRNAs (miRNAs/miRs) are non-protein-encoding small RNAs that are encoded in the genome, and negatively regulate target gene expression at the post-transcriptional level $(8,9)$. A number of studies have demonstrated that the importance of these small RNAs in disease initiation and progression was dependent on the regulation of distinct disease-specific signal transduction pathways $(10,11)$. Increasing evidence has indicated that several miRNAs act as a novel class of regulators in certain cardiovascular diseases (12). Notably, the dysregulation of certain miRNAs regulates various fibrosis-associated proteins to influence the development and progression of cardiac fibrosis $(13,14)$. It has been reported that miR-155, one member of the small RNA family, has an extensive and close association with circulatory system diseases (15). A previous report indicated that miR-155 acts as a key mediator of cardiac injury and inflammation in atherosclerosis by repressing B-cell CLL/lymphoma 6 in macrophages (16). Additionally, inhibition of miR-155 in mouse cardiomyocytes was associated with protection from cardiac hypertrophy, and the repression of endogenous jumonji and 
AT-rich interaction domain containing 2 (Jarid2) in isolated cardiomyocytes partially rescued the effect of miR-155 loss, as previously reported (17). In addition, miR-155 expression in macrophages also promotes cardiac inflammation, hypertrophy and failure in response to Ang II by regulating suppressor of cytokine signaling 1 (SOCS1) (18). Furthermore, a recent study demonstrated the proinflammatory effects of miR-155 in the promotion of liver fibrosis induced by alcohol or carbon tetrachloride and alcohol-induced steatohepatitis (19).

However, data concerning the effect of miR-155 on cardiac fibrosis, particularly in cardiac fibroblasts, remains limited. The present study reported that miR-155, a frequently upregulated miRNA in hypertrophic hearts, was able to increase the excessive deposition of ECM proteins during the process of cardiac remodeling. The results indicated that manipulating the expression of miR-155 may be a promising therapeutic strategy for cardiac fibrosis.

\section{Materials and methods}

Animal studies. Male miR-155\% mice from a C57BL/6 background were purchased from Jackson Laboratory (Ben Harbor, ME, USA). Male wild-type (WT) C57BL/6 mice were purchased from Beijing HFK Bioscience Co., Ltd (Beijing, China). All experiments were conducted with the approval of the Animal Care Committee of the Union Hospital of Tongji Medical College, Huazhong University of Science and Technology (Wuhan, China). Healthy adult male miR-155 ${ }^{-/}$mice $(n=80)$ and C57BL/6 mice $(n=120)$ used in the present study were kept under standard animal room conditions (temperature, $21 \pm 1^{\circ} \mathrm{C}$; humidity, 50-60\%; $0.03 \% \mathrm{CO}_{2}$; $12 \mathrm{~h}$ for light and $12 \mathrm{~h}$ for dark) with food and water prior to experiments. The cardiac hypertrophy model was induced in male $\mathrm{miR}-155^{-/}$and WT C57Bl/6J mice (age, 10-12 weeks; weight, $20-25 \mathrm{~g})$ by Ang II (1.5 $\mu \mathrm{g} / \mathrm{g} / \mathrm{day}$; Sigma-Aldrich; Merck KGaA, Darmstadt, Germany) infusion for 4 consecutive weeks using subcutaneously implanted ALZET ${ }^{\circledR} 2004$ minipumps. Sham groups (control) received an equivalent dose of PBS instead of Ang II. Animal models were divided into different groups as described in Table I, and mice $(n=6-8)$ were randomly assigned to each of the indicated treatment groups. Food and water were available ad libitum throughout the duration of experiment. When the drug was depleted after 4 weeks, some mice were sacrificed, however, others continued to receive treatment for an additional 4 weeks. All animals were anaesthetized intraperitoneally with sodium pentobarbital $(50 \mathrm{mg} / \mathrm{kg})$ and exsanguinated via a retro-orbital venous puncture after 4 or 8 weeks of treatment. Hearts and spleens were harvested rapidly from the euthanized mice following rapid cervical dislocation.

1-3-day-old neonatal male WT C57BL/6 mice $(n=100)$ were purchased from Experimental Animal Center of Wuhan University (Wuhan, China) and were kept in the pathogen-free room in the experimental animal center (Tongji Medical College of Huazhong University of Science and Technology) under the same housing conditions as aforementioned.

Assessment of cardiac function. The cardiac function of mice was evaluated noninvasively by echocardiography performed with a Vevo 1100 imaging system (FUJIFILM VisualSonics,
Inc., Toronto, ON, Canada) equipped with an MS400 (18-38 MHz) phased-array transducer after 4 and 8 weeks of Ang II treatment, as previously described (20). Left ventricular diastole diameter (LVDd) and interventricular septum diastolic thickness (IVS) were measured, and left ventricular ejection fraction (LVEF) and left ventricular fractional shortening (LVFS) were calculated from measured recordings. The sonographer was blinded to the randomization of mice. Every 2 weeks, the systolic blood pressure was determined via tail-cuff plethysmography by using a non-invasive blood pressure controller and PowerLab system.

Obtaining peripheral blood mononuclear cells (PBMCs). Peripheral blood samples were collected via a retro-orbital venous puncture following anesthetizing the mice, as aforementioned. PBMCs were separated from erythrocytes by density centrifugation at $700 \mathrm{x} \mathrm{g}, 18^{\circ} \mathrm{C}$ for $20 \mathrm{~min}$, using Ficoll ${ }^{\circledR}$ PM 400 Histopaque ${ }^{\circledR}-1077$ (Sigma-Aldrich; Merck $\mathrm{KGaA}$ ). Isolated PBMCs were prepared for RNA analysis.

Cardiac fibroblast isolation and culture. Mouse neonatal cardiac fibroblasts were prepared from the hearts of 1-3-day-old neonatal male WT C57BL/6 mice, which were finely minced and placed together in $0.25 \%$ trypsin, as described previously $(21,22)$. The resuspension was plated onto culture flasks with Dulbecco's modified Eagle's medium (DMEM; Sigma-Aldrich; Merck KGaA) containing 10\% fetal bovine serum (Gibco; Thermo Fisher Scientific, Inc., Waltham, MA, USA) for $60 \mathrm{~min}$ at $37^{\circ} \mathrm{C}$ in humidified air with $5 \% \mathrm{CO}_{2}$, which allowed for preferential attachment of fibroblasts to the bottom of the culture flasks. Flasks were washed twice with PBS to detach the weakly attached and non-adherent cells and the medium was changed. Cardiac fibroblasts were cultured in Dulbecco's modified Eagle's medium (DMEM; Sigma-Aldrich; Merck KGaA) containing $10 \%$ fetal bovine serum (Gibco; Thermo Fisher Scientific, Inc.) at $37^{\circ} \mathrm{C}$ in humidified air with $5 \% \mathrm{CO}_{2}$. Cells were removed into 24 - or 6 -well plates $\left(1 \times 10^{6}\right.$ cells $\left./ \mathrm{ml}\right)$ for experimental preparation with DMEM containing $1 \%$ fetal bovine serum for $12 \mathrm{~h}$ at $37^{\circ} \mathrm{C}$. Subsequently, the cells were treated with Ang II $(1 \mu \mathrm{M})$ and controls were treated with the same dose of PBS for $48 \mathrm{~h}$ at $37^{\circ} \mathrm{C}$ in a humidified $5 \% \mathrm{CO}_{2}$ atmosphere (22).

Cell transfection. Cardiac fibroblasts were cultured in 24-well plates until they reached $50-80 \%$ confluence, as previously described (23). Cell transfection was performed using a riboFEC CP Transfection kit (Guangzhou RiboBio Co., Ltd., Guangzhou, China), according to the manufacturer's protocol. Cardiac fibroblasts were transfected with double-chain agomiR-155 (mature sequence, UUAAUGCUAAUUGUG AUAGGGGU; complementary sequence, AAUUACGAU UAACACUAUCCCCA) or agomir negative control for miR-155 overexpression, and single-chain antagomiR-155 (complementary sequence, AAUUACGAUUAACACUAU CCCCA) or antagomir negative control for miR-155 inhibition. The agomir and antagomir negative control were the same (UUUGUACUACACAAAAGUACUG). Cardiac fibroblasts were transfected with agomiR-155 or an agomir negative control at a concentration of $20 \mathrm{nM}$, and antagomiR-155 or an antagomir negative control at a final concentration of $100 \mathrm{nM}$. 
Table I. Characteristics of mice following 4 and 8 weeks of Ang II infusion.

A, Characteristics of mice following 4 weeks of Ang II infusion

\begin{tabular}{lccc}
\hline Group & Number & Body weight, & H/B, mg/g \\
\hline WT-sham & 8 & $26.5 \pm 1.2$ & $4.5 \pm 0.4$ \\
KO-sham & 6 & $26.1 \pm 1.3$ & $4.3 \pm 0.6$ \\
WT-Ang II & 8 & $28.6 \pm 1.6$ & $6.5 \pm 0.6^{\mathrm{a}}$ \\
KO-Ang II & 6 & $28.3 \pm 1.6$ & $5.8 \pm 0.3^{\mathrm{b}, \mathrm{c}}$ \\
\hline
\end{tabular}

B, Characteristics of mice following 8 weeks of Ang II infusion

\begin{tabular}{lccc}
\hline Group & Number & Body weight, & $\mathrm{H} / \mathrm{B}, \mathrm{mg} / \mathrm{g}$ \\
\hline WT-sham & 8 & $29.3 \pm 1.1$ & $4.6 \pm 0.8$ \\
KO-sham & 6 & $28.9 \pm 1.9$ & $4.5 \pm 0.7$ \\
WT-Ang II & 8 & $30.5 \pm 1.7$ & $6.7 \pm 0.4^{\mathrm{a}}$ \\
KO-Ang II & 6 & $30.1 \pm 1.4$ & $6.5 \pm 0.5^{\mathrm{b}}$ \\
\hline
\end{tabular}

${ }^{\mathrm{a}} \mathrm{P}<0.05$ vs. WT-sham; ${ }^{\mathrm{b}} \mathrm{P}<0.05$ vs. KO-sham; ${ }^{\mathrm{P}}<0.05$ vs. WT-Ang II. Ang, angiotensin; H/B, heart weight/body weight; WT, wild-type; KO, knockout.

Subsequently, cells were incubated for $6 \mathrm{~h}$ at $37^{\circ} \mathrm{C}$, the medium was changed and cells were cultured with Ang II stimulation (100 $\mathrm{nmol} / \mathrm{l})$ for $48 \mathrm{~h}$ in DMEM containing 5\% fetal bovine serum at $37^{\circ} \mathrm{C}$ in humidified air with $5 \% \mathrm{CO}_{2}$. Cells were harvested after $48 \mathrm{~h}$. Second passage cells were used in all experiments. All agents for transfection were purchased from Guangzhou RiboBio Co., Ltd.

Western blot analysis. Total protein was isolated from heart tissue and cultured cardiac fibroblasts, as previously described (24). Protein concentration was quantified using a BCA protein assay kit (Pierce; Thermo Fisher Scientific, Inc.) according to the manufacturer's protocol. Briefly, protein samples (50 $\mu \mathrm{g} / \mathrm{lane})$ were subjected to SDS-PAGE (10\% gel) and transferred to polyvinylidene difluoride membranes. The blots were blocked with $5 \%$ nonfat milk in TBS containing $0.05 \%$ Tween-20 (TBST) for $2 \mathrm{~h}$ at room temperature, and subsequently probed with specific primary antibodies against $\alpha$-SMA (1:1,000; YM3364; ImmunoWay Biotechnology Company, Plano, TX, USA), type I collagen (1:1,000; YT6135 ImmunoWay Biotechnology Company) and GAPDH (1:1,000; YM1038; ImmunoWay Biotechnology Company) at $4^{\circ} \mathrm{C}$ overnight. The membranes were washed with TBST and incubated with secondary horseradish peroxidase-conjugated antibodies $(1: 3,000 ; 14708 \mathrm{~S}$; Cell Signaling Technology, Inc., Danvers, MA, USA) for $2 \mathrm{~h}$ at room temperature. Finally, the protein bands were washed and developed with enhanced chemiluminescence (ECL) kit (Thermo Fisher Scientific, Inc.) and semiquantitatively analyzed using densitometric methods in each group with Quantity One software (version 4.62; Bio-Rad Laboratories, Inc., Hercules, CA, USA). The results were expressed as fold changes by normalizing the data to the control values.

Reverse transcription-quantitative polymerase chain reaction (RT-qPCR) analysis. Total RNA from cardiac fibroblasts,
PBMCs and heart and spleen tissues was isolated using TRIzol reagent (Invitrogen; Thermo Fisher Scientific, Inc.), and was reverse transcribed into cDNA by using a PrimeScript RT reagent kit at $37^{\circ} \mathrm{C}$ for 15 min (Takara Bio, Inc., Otsu, Japan), according to the manufacturer's protocol. The $10 \mu \mathrm{l}$ PCR mixture contained $1 \mu \mathrm{g}$ total cDNA and 5 pmol each of the primers. The primers for mRNAs are listed in Table II. GAPDH was used as an internal standard. All reactions were performed with SYBR Premix Ex Taq II (Takara Bio, Inc.) and incubated in a 7500 Real-Time PCR system (Applied Biosystems; Thermo Fisher Scientific, Inc.) in a 96-well plate, according to the manufacturer's protocol. For miR-155 detection, the following thermocycling conditions were used: An initial predenaturation step at $50^{\circ} \mathrm{C}$ for $2 \mathrm{~min}$, followed by 40 cycles of denaturation at $95^{\circ} \mathrm{C}$ for $10 \mathrm{~min}$ and annealing at $60^{\circ} \mathrm{C}$ for $1 \mathrm{~min}$. For other factor detection, the thermocycling conditions were as follows: An initial predenaturation step at $94^{\circ} \mathrm{C}$ for $5 \mathrm{~min}$, followed by 40 cycles of denaturation at $95^{\circ} \mathrm{C}$ for $30 \mathrm{sec}$, annealing at $60^{\circ} \mathrm{C}$ for $30 \mathrm{sec}$ and extension at $72^{\circ} \mathrm{C}$ for $20 \mathrm{sec}$. Each sample was repeated at least three times. The quantitative assessment of specific miR-155 level was measured using standard protocols, as previously described (23). The expression levels of target genes relative to endogenous controls was quantified by comparative quantitation cycle method (24) The primers used in the present study were purchased from Guangzhou RiboBio Co., Ltd. The primer sequences used are presented in Table II. Data were expressed as the fold change compared with the control.

Histopathological experiments. The left ventricle was fixed in $4 \%$ formaldehyde for $48 \mathrm{~h}$ at $4^{\circ} \mathrm{C}$ and embedded in paraffin. Adjacent paraffin-embedded left ventricle sections $(5 \mu \mathrm{m})$ were cut for hematoxylin and eosin (HE) and Masson's trichrome staining. In brief, paraffin-embedded tissue sections were stained with dimethylbenzene for $15 \mathrm{~min}$ 
Table II. Primer sequences for reverse transcription-quantitative polymerase chain reaction.

\begin{tabular}{lll}
\hline Gene & \multicolumn{1}{c}{ Forward primer, 5'-3' } & \multicolumn{1}{c}{ Reverse primer, 5'-3' } \\
\hline SOCS1 & CTGCGGGCTTCTATTGGGGAC & AAAAGGCAGTCGAAGGTCTCG \\
Collagen I & GACTGGCAACCTCAAGAAGG & GACTGTCTTGCCCCAAGTTC \\
TGF- $\beta 1$ & CTCCCGTGGCTTCTAGTGC & GCCTTAGTTTGGACAGGATCTG \\
GAPDH & AGGTCGGTGTGAACGGATTTG & TGTAGACCATGTAGTTGAGGTCA \\
$\alpha-$ SMA & GTCCCAGACATCAGGGAGTAA & TCGGATACTTCAGCGTCAGGA \\
SHIP1 & GCCCCTGCATGGGAAATCAA & TGGGTAGCTGGTCATAACTCC \\
SMAD3 & CACGCAGAACGTGAACACC & GGCAGTAGATAACGTGAGGGA \\
U6 & CTCGCTTCGGCAGCACA & AACGCTTCACGAATTTGCGT \\
MiR-155 & TGCCTCCAACTGACTCCTAC & GCCAGCAGAATAATACGAC
\end{tabular}

SOCS1, suppressor of cytokine signaling 1; TGF, transforming growth factor; SMA, smooth muscle actin; SHIP, Src homology 2-containing inositol phosphatase.

at room temperature, then stained with hematoxylin for 10-15 min and washed with water prior to being stained with $0.5 \%$ eosin for $30 \mathrm{sec}-1 \mathrm{~min}$ then washed with water at room temperature to demonstrate distinct colors in the nucleus and cytoplasm. For Masson's trichrome, paraffin-embedded tissue sections were stained with Regaud hematoxylin for 5-10 min at room temperature, then stained with masson acid complex red liquid for $5 \mathrm{~min}$ and $0.2 \%$ glacial acetic acid aqueous solution for $30 \mathrm{sec}$ at room temperature then washed in water, and subsequently stained with toluidine blue for $5 \mathrm{~min}$ at room temperature. The HE Staining kit and Masson's trichrome Staining kit used in this study were purchased from Wuhan Goodbio Technology Co., Ltd. (Wuhan, China), according to manufacturer's protocol. Heart sections were stained with HE and Masson's trichrome for the assessment of inflammatory cell areas and collagen volume fraction with $\mathrm{HE}$ and Masson procedures, respectively (25). A BX51TF microscope (magnification, x40, 100, 200 and 400; Olympus Corporation, Tokyo, Japan) was used to observe pathological change areas. Image-Pro Plus 6.703 software (Media Cybernetics, Inc., Rockville, MD, USA) was used to calculate fibrosis areas and the statistical analysis.

Statistical analysis. Data are presented as the mean \pm standard error of the mean. All analyses were performed using GraphPad Prism software (version 5.0; GraphPad Software, Inc., La Jolla, CA, USA). Student's test was performed for comparisons between two groups and one-way analysis of variance followed by Bonferroni's post-hoc test was performed among multiple groups. $\mathrm{P}<0.05$ was considered to indicate a statistically significant difference.

\section{Results}

miR-155 is expressed in mouse hearts and its expression is induced in cardiac remodeling. Previous studies have reported that the expression of miR-155 is ubiquitous in adult mouse tissues, and is enriched in T lymphocytes and infiltrating macrophages (26-28). The present study investigated the expression of miR-155 in the heart, PBMCs and spleen of adult mice. As demonstrated in Fig. 1A-C, miR-155 was expressed in adult mouse tissues. In addition, the expression of miR-155 was increased during the process of cardiac remodeling induced by treatment with Ang II for 4 and 8 weeks. These results indicate that miR-155 is expressed in the hearts of adult mice and that its expression is regulated during cardiac remodeling.

miR-155 deficiency improves cardiac function in mice. As miR-155 was significantly upregulated in mouse hearts during cardiac remodeling induced by Ang II, miR- $155^{-/}$and WT mice were subjected to Ang II infusion for 4 and 8 weeks to investigate whether the absence of miR-155 influences Ang II-induced cardiac remodeling. As demonstrated in Fig. 2A, Ang II induced heart hypertrophy in both $\mathrm{miR}-155^{-/-}$and WT mice, as evidenced by an increase in the size of hearts compared with sham mice. However, the size of the heart in $\mathrm{miR}-155^{-/}$mice appeared substantially smaller compared with WT mice following Ang II infusion. The development of cardiac hypertrophy in the hearts of WT mice and the repression of hypertrophic growth in miR-155/- mice following Ang II infusion are supported by differences in the heart weight/body weight ratio (Table I). The mean arterial pressure (MAP) of mice in each group at different time points was also measured, and similar changes in the MAP were observed in WT compared with miR-155/- mice, with marginally higher MAP in the WT group throughout treatment (Fig. 2B).

Echocardiography was performed to determine cardiac function at different time points in each group (Fig. 2C). As demonstrated in Fig. 2D-G, cardiac dysfunction, as demonstrated by significantly decreased LVEF and LVFS compared with the sham group at 8 weeks, was observed in the Ang II-treated WT group. However, LVEF and LVFS were significantly higher at 8 weeks in the miR-155/- Ang II-treated group compared with the WT Ang II-treated group. In addition, increases in LVDd and IVS were more pronounced in in the Ang II-treated WT group compared with the Ang II-treated miR-155 $5^{-/}$mice at 4 and 8 weeks. Combined, these results indicate that the absence of miR-155 in mice markedly improved cardiac function during the process of Ang II-induced cardiac remodeling. 
A

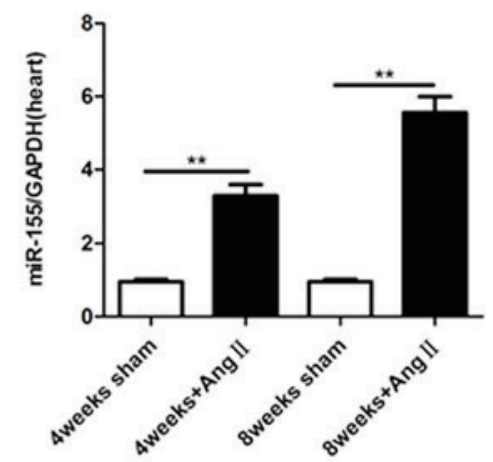

B

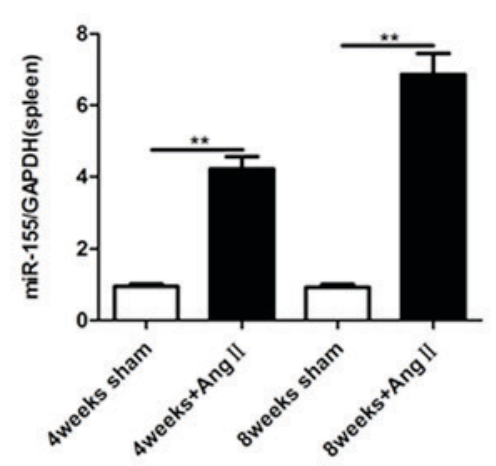

C

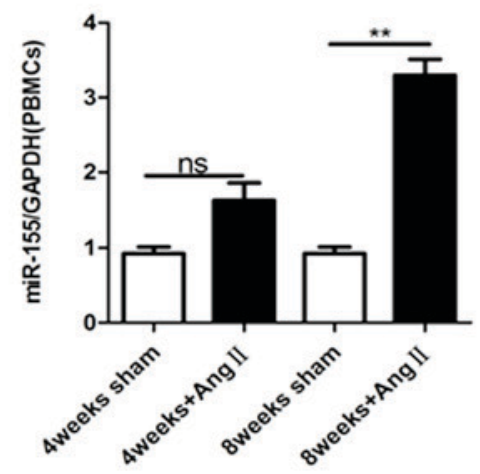

Figure 1. miR-155 is induced by Ang II treatment in adult C57BL/6 mice. The expression of miR-155 in the (A) heart, (B) spleen and (C) PBMCs of WT adult mice following Ang II infusion for 4 and 8 weeks, as quantified by reverse transcription-quantitative polymerase chain reaction. Data are presented as the mean + standard error of the mean. ${ }^{*} \mathrm{P}<0.05$ and ${ }^{* * *} \mathrm{P}<0.01$, as indicated. miR, microRNA; Ang, angiotensin; PBMCs, peripheral blood mononuclear cells; WT, wild-type; NS, not significant.

A

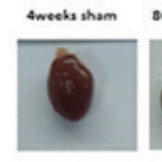

Ko

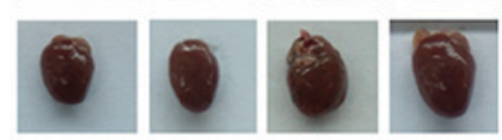

B

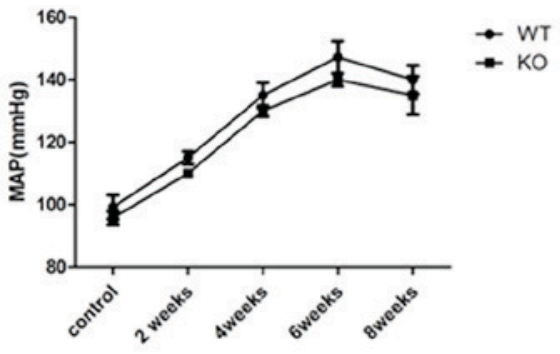

C

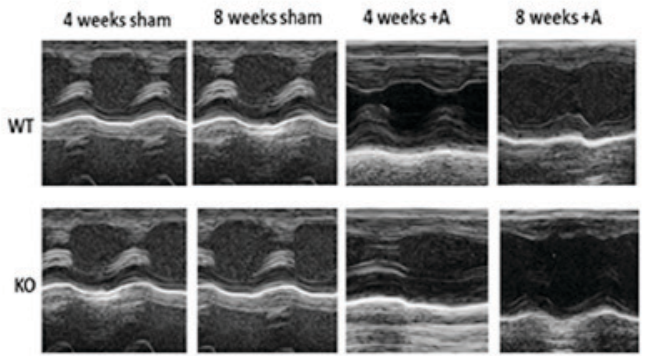

D

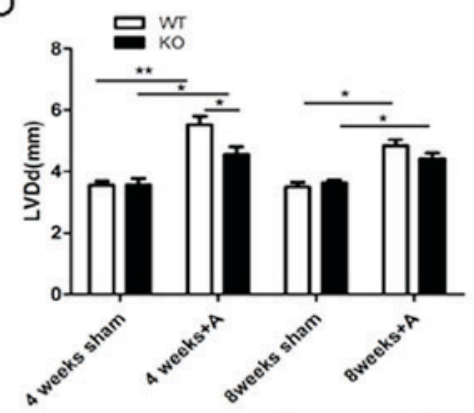

$\mathrm{E}$

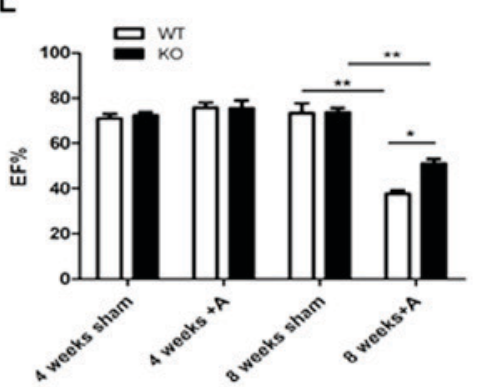

$\mathrm{F}$

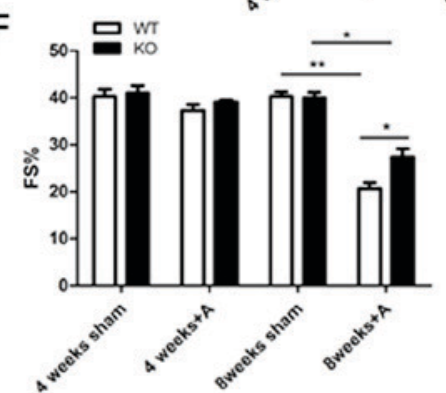

G

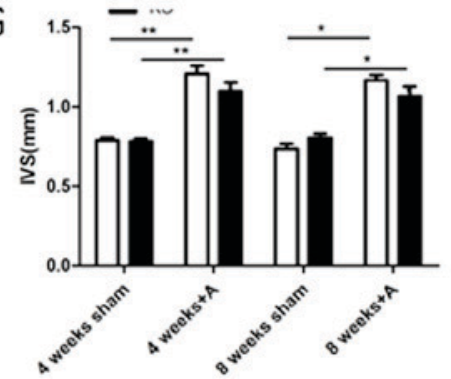

Figure 2. Absence of miR-155 ameliorates the cardiac function following Ang II treatment. (A) Representative images of whole hearts in WT and miR-155 KO groups with or without Ang II treatment. (B) MAP of mice in WT and miR-155 KO groups at different time points during Ang II treatment. (C) Representative M-mode echocardiograms obtained from WT-sham, KO-sham, WT-Ang II and KO-Ang II mice. Echocardiographic measurements of (D) LVDd, (E) LVEF, (F) LVFS and (G) IVS were recorded in WT and KO-sham and Ang II mice. Data are presented as the mean \pm standard error of the mean. ${ }^{*} \mathrm{P}<0.05$ and ${ }^{* *} \mathrm{P}<0.01$, as indicated. miR, microRNA; Ang, angiotensin; WT, wild-type; KO, knockout; MAP, mean arterial pressure; LVDd, left ventricular diastole diameter; LVEF, left ventricular ejection fraction; LVFS, left ventricular fractional shortening; IVS, interventricular septum diastolic thickness; A, Ang II.

miR-155 deficiency alleviates cardiac fibrosis. To investigate the potential effects of miR-155 on cardiac fibrosis, differences in the severity of fibrosis based on the infiltration of inflammatory cells and fibrosis area of the left ventricle in miR-155 $1 /$ mice and WT mice were examined. HE and Masson's trichrome staining were performed to assess 

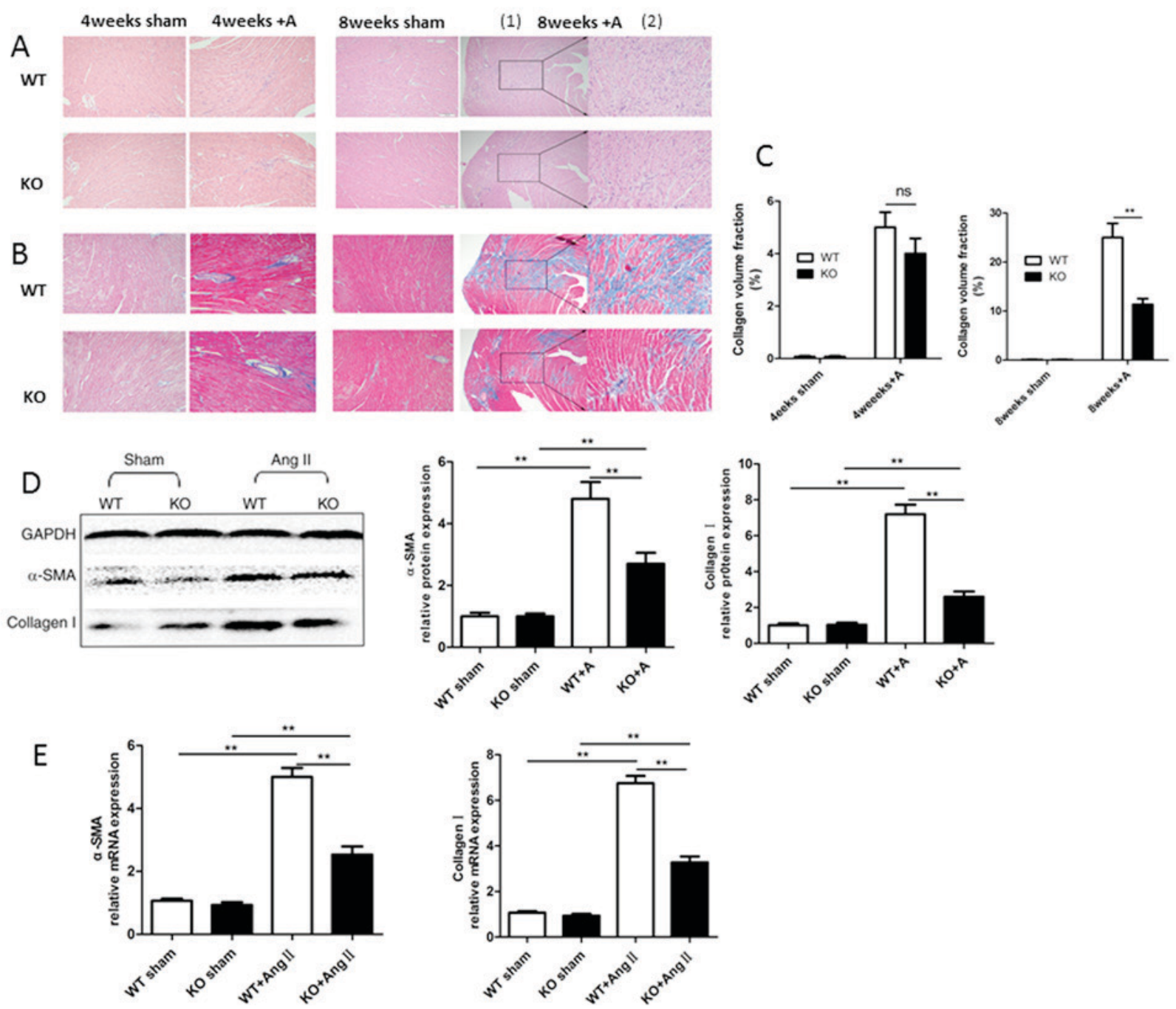

Figure 3. miR-155 deficiency alleviates Ang II-induced cardiac fibrosis (A) Hematoxylin and eosin-stained sections of the left ventricles in miR-155 WT and KO mice. (B) Representative Masson's trichrome staining of cross-sections of left ventricles. Panels A and B: Magnification, x200 for 4 weeks (sham and $+\mathrm{A}$ ) and $\mathrm{x} 100$ for 8-weeks [sham and $+\mathrm{A}(1)$ ]; $\mathrm{x} 400$ for 8-weeks+A(2). (C) Collagen volume fraction in miR-155 WT and KO mice. (D) Representative western blot for $\alpha$-SMA and collagen I protein expression levels in heart tissues following Ang II treatment for 8 weeks. (E) mRNA expression levels of $\alpha$-SMA and collagen I in each group at 8 weeks. Data are presented as the mean + standard error of the mean. ${ }^{*} \mathrm{P}<0.05$ and ${ }^{* * *} \mathrm{P}<0.01$, as indicated. miR, microRNA; Ang, angiotensin; WT, wild-type; KO, knockout; SMA, smooth muscle actin; A, Ang II.

inflammatory cell areas and collagen volume fractions, respectively. As demonstrated in Fig. 3A and B, inflammatory cell infiltration was markedly reduced in miR-155 $5^{-/}$mice with Ang II treatment compared with Ang II-treated WT mice, and the interstitial fibrotic response was also reduced in miR-155 ${ }^{-1-}$ compared with WT mice, particularly at 8 weeks. In addition, the fibrosis areas in miR-155 ${ }^{--}$and WT groups were calculated as the collagen volume fraction using Image-Pro Plus 6.703 software, as demonstrated in Fig. 3C.

Myofibroblasts are the primary source of ECM and have a critical role in cardiac fibrosis, they are characterized by the presence of a microfilamentous contractile apparatus enriched with $\alpha$-SMA. Therefore, the expression of $\alpha$-SMA and collagen I was determined to clarify whether miR-155 has a direct role in cardiac fibrosis. As demonstrated in Fig. 3D and $\mathrm{E}$, the protein and mRNA expression levels of $\alpha$-SMA and collagen I were decreased in miR-155 $5^{--}$mice compared with WT mice following 8 weeks of Ang II treatment. These results indicate that miR-155 may be involved in cardiac fibrotic remodeling, and may increase collagen production in Ang II-induced cardiac remodeling.

miR-155 induces a profibrotic myofibroblast phenotype in isolated cardiac fibroblasts. Cardiac fibroblasts have important functions in reparative and detrimental fibrotic responses during the process of cardiac remodeling. To determine whether miR-155 is involved in cardiac fibroblasts, mouse neonatal cardiac fibroblasts were prepared from neonatal C57BL/6 mice. The expression of miR-155 was low in cardiac fibroblasts, however, the expression was significantly induced by Ang II stimulation for $48 \mathrm{~h}$ (Fig. 4A). Similarly, cultured mouse neonatal cardiac fibroblasts treated with Ang II also exhibited increased expression of $\alpha$-SMA compared with control cells (Fig. 4B). To further confirm whether miR-155 was involved in the expression of $\alpha$-SMA, gain- and loss-of-function studies on the expression of miR-155 were performed by transfection with agomiR-155 and antagomiR-155, respectively. miR-155 expression was significantly elevated 

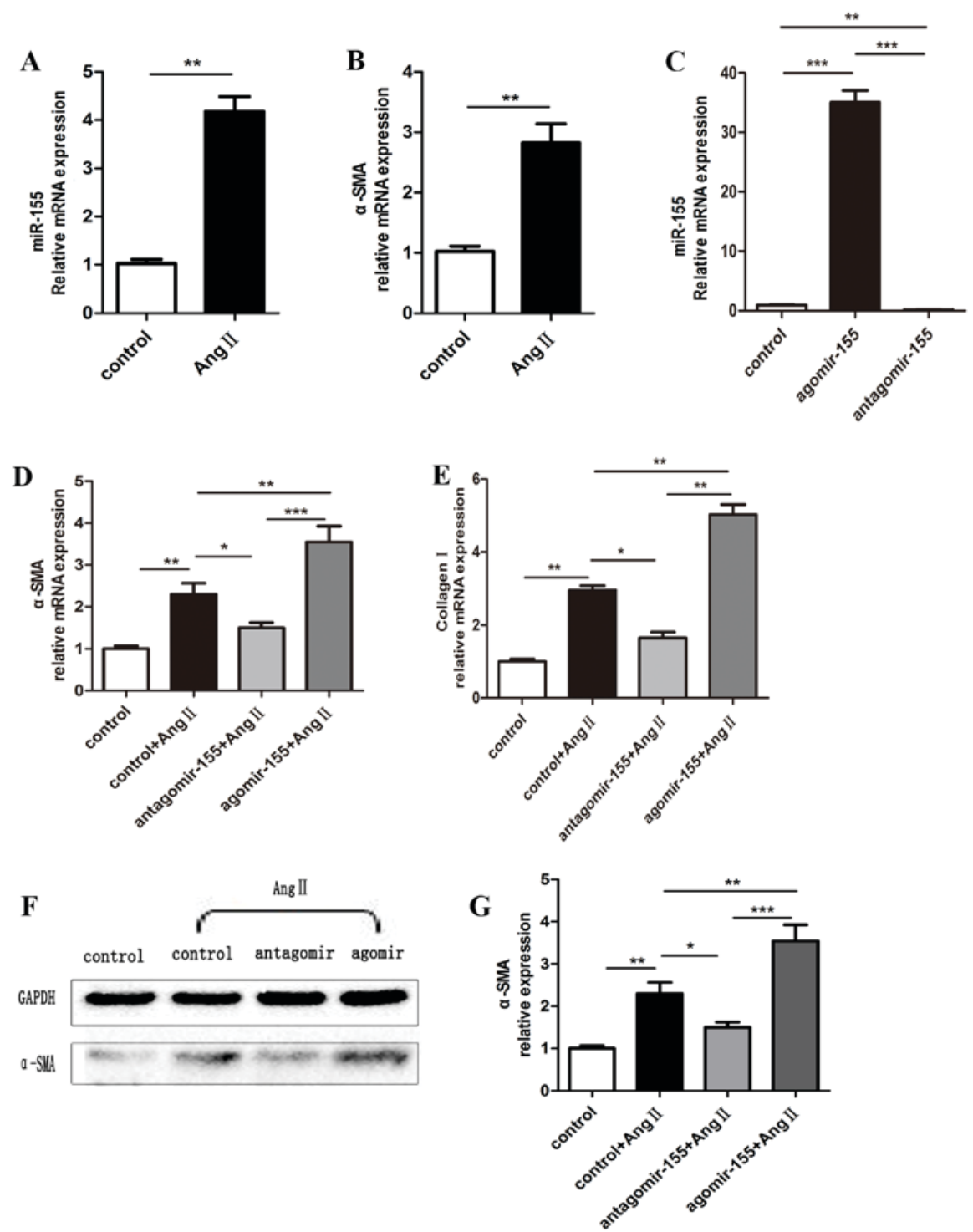

Figure 4. miR-155 regulates fibroblast to myofibroblast transformation in cardiac fibroblasts. The mRNA expression levels of (A) miR-155 and (B) $\alpha$-SMA in cardiac fibroblasts following Ang II stimulation. (C) miR-155 expression in cells in each group following transfection with miR-155 agomir or antagomir, as determined by reverse transcription-quantitative polymerase chain reaction. mRNA expression levels of (D) $\alpha$-SMA and (E) collagen I in each group of cardiac fibroblasts. (F) Representative image of western blotting for $\alpha$-SMA protein expression levels in cardiac fibroblasts from each group. (G) Quantified protein levels of $\alpha$-SMA in each group. Data are presented as the mean + standard error of the mean. ${ }^{*} \mathrm{P}<0.05,{ }^{* * *} \mathrm{P}<0.01$ and ${ }^{* * * *} \mathrm{P}<0.001$, as indicated. miR, microRNA; SMA, smooth muscle actin; Ang, angiotensin.

in cardiac fibroblasts transfected with agomiR-155, whereas levels were suppressed in antagomiR-155-treated cells, compared with the control-transfected cells (Fig. 4C). Notably, as demonstrated in Fig. 4D-G, forced expression of miR-155, using agomiR-155, increased the expression levels of $\alpha$-SMA and collagen I in Ang II-treated fibroblasts compared with the control-transfected group, while the opposite effects were observed in antagomiR-155-treated cells. In conclusion, these results indicate that miR-155 may contribute to cardiac fibrotic remodeling by inducing fibroblast to myofibroblast transformation.

SOCS1 is involved in the process of miR-155-regulated fibrosis. Numerous miR-155 target genes have previously been described (29). Direct anti-inflammatory miR-155 targets include SOCS1 and Src homology 2-containing inositol phosphatase-1 (SHIP1). The present study demonstrated that, in the absence of miR-155, SOCS1 expression was elevated in Ang II-treated mice compared with WT Ang II-treated mice (Fig. 5A), whereas SHIP1 was not significantly induced in miR-155 $5^{-1}$ mice with Ang II treatment compared with WT mice with Ang II treatment (Fig. 5B). Therefore, we hypothesized that SOCS1, which functions as a negative regulator of cytokine signaling, may be involved in miR-155-regulated cardiac fibrosis. The results of the current study also indicated that increased SOCS1 levels in Ang II-treated miR-155 ${ }^{-/}$mice were associated with diminished profibrotic transforming growth factor (TGF)- $\beta 1 /$ SMAD3 signaling, compared with WT Ang II-treated mice (Fig. 5C and D). In addition, the results in Fig. 3E indicate that a reduction in the expression of collagen I may also be associated with increased SOCS1 expression, as collagen expression was reduced in Ang 

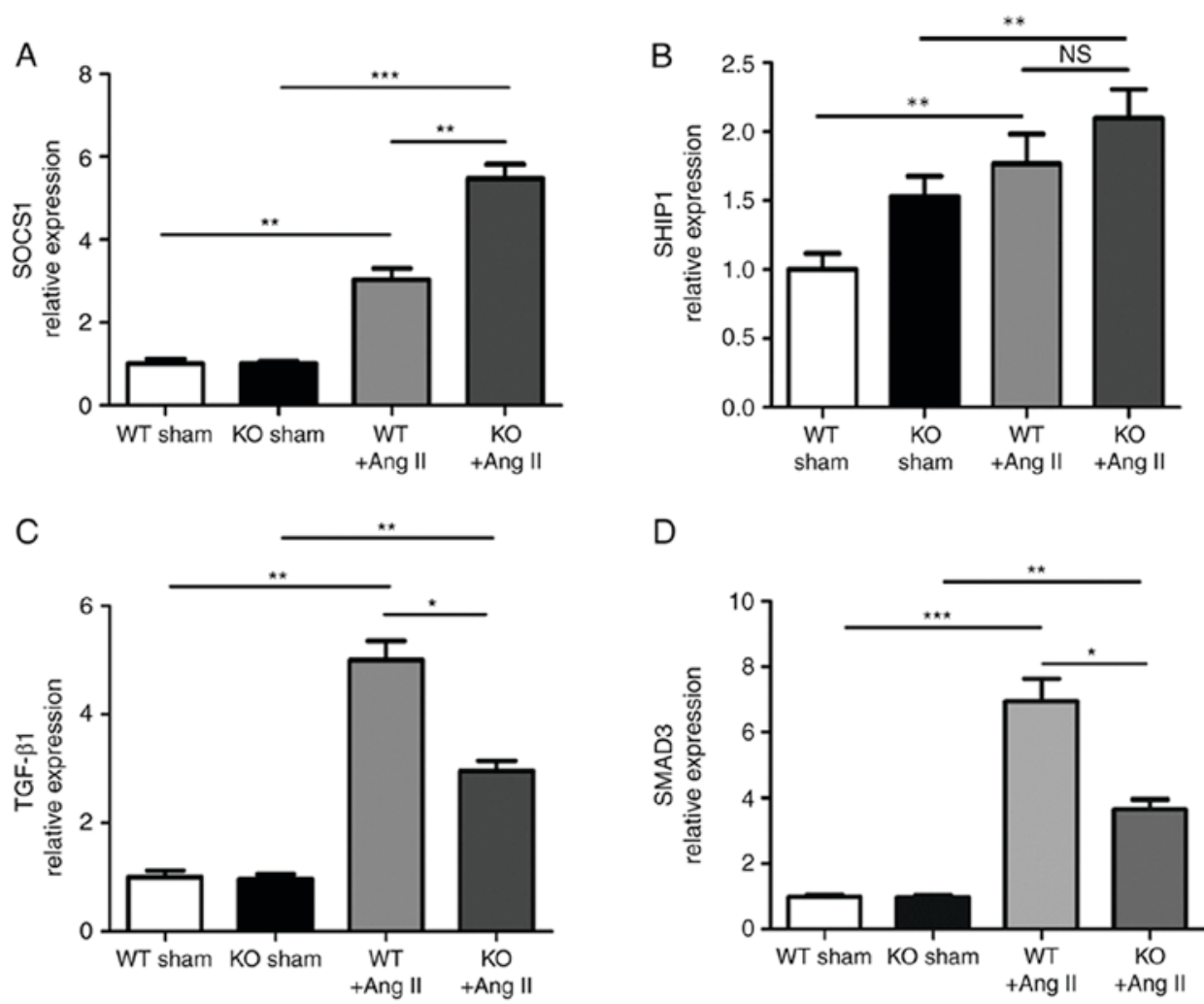

Figure 5. SOCS1 was involved in miR-155-regulated cardiac fibrosis. mRNA expression levels of (A) SOCS1 and (B) SHIP1, which are direct target miR-155 targets, in KO and WT mice heart tissues at 8 weeks. (C and D) mRNA expression levels of TGF- $\beta 1$ and SMAD3 in heart tissues of KO and WT mice. Data are presented as the mean + standard error of the mean. ${ }^{*} \mathrm{P}<0.05,{ }^{* *} \mathrm{P}<0.01$ and ${ }^{* * * *} \mathrm{P}<0.001$, as indicated. SOCS1, suppressor of cytokine signaling 1 ; miR, microRNA; SHIP1, Src homology 2-containing inositol phosphatase; KO, knockout; WT, wild-type; TGF, transforming growth factor.

II-treated miR-155 ${ }^{-/}$mice compared with Ang II-treated WT mice, while SOCS1 expression was increased. These results indicate that miR-155 deficiency may inhibit profibrotic TGF-31/SMAD-3 signaling via interactions with SOCS1.

\section{Discussion}

It is evident that cardiac fibrosis, characterized by excessive ECM deposition, seriously limits cardiac systolic and diastolic function (30). Various growth and inflammatory factors are reported to be involved in this process, including certain miRNAs $(13,14,31)$. Previous studies have reported that miR-155 has an important role in various inflammatory heart diseases, including cardiac hypertrophy, myocarditis, atherosclerosis and heart failure $(15,16,18)$. Despite this, it is generally accepted that fibroblasts are critically involved in the reparative response and the pathogenesis of cardiac remodeling. However, direct evidence for a role of miR-155 in Ang II-induced cardiac fibrotic remodeling is limited. The present

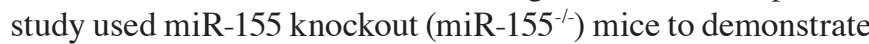
that miR-155 enhances fibrosis in Ang II-induced cardiac remodeling. Furthermore, gain- and loss-of-function studies on the expression of miR-155 were performed in cardiac fibroblasts to demonstrate that miR-155 is involved in the induction of fibroblast to myofibroblast transformation.

It is established that certain miRNAs are expressed abnormally in a variety of diseases (32). As certain miRNAs have important roles in response to stress signals, it may be hypothesized that miRNAs may be involved in modulating the progression from adaptive to pathological cardiac remodeling by acting alone or in combination. The results of the present study demonstrated that the expression of miR-155 was upregulated in the damaged heart tissue of mice, and miR-155 $\%$ mice exhibited relatively mild heart damage compared with WT mice in response to Ang II treatment. This result is consistent with the results of previous studies, which demonstrated that miR-155 expression promoted cardiac inflammation, hypertrophy and failure in response to pressure overload $(17,18)$. However, the existing literature concerning the role of miR-155 in cardiac fibrotic remodeling is conflicting. Heymans et al (18) and confirmed that absence of miR-155 reduced pressure overload-induced cardiac hypertrophy and inflammation, while the cardiac fibrotic response remained intact. In addition, their results indicated that pressure overload-induced fibrotic remodeling may be independent of macrophage miR-155 function, or fibrotic remodeling in mouse hearts may not be solely dependent on macrophage signaling. Furthermore, another study concluded that loss of miR-155 substantially eliminated the increase in cardiomyocyte size in transverse aortic constriction-induced hypertrophic mice, and cardiac fibrosis was markedly suppressed in the hearts of miR-155 $15^{--}$mice. They reported that endogenous miR-155 may suppress cardiomyocyte hypertrophy by targeting Jarid2 in isolated cardiomyocytes, however, the mechanism of miR-155-induced cardiac fibrosis was not reported (17). However, despite these results, the 
mechanisms of miR-155-regulated fibrotic remodeling, particularly in cardiac fibroblasts, remain unclear. By contrast, the present study overexpressed and inhibited miR-155, by using miR-155 agomir and antagomirs, in cardiac fibroblasts to demonstrate that miR-155 may partially affect cardiac fibrosis by inducing fibroblast to myofibroblast transformation. The results of the current study also demonstrated that the expression of $\alpha$-SMA increased with increasing miR-155 expression in Ang II-treated hearts and cardiac fibroblasts, indicating that miR-155 may be involved in fibroblast to myofibroblast transformation. Unfortunately, persistent myofibroblast activation and the resultant increase in fibrous tissue produced may cause progressive adverse myocardial remodeling.

The mechanisms by which miRNAs regulate cardiac fibrosis have been attributed to the alteration of certain signaling pathways in the pathological process of fibrotic growth (33). It is established that certain inflammatory factors have important functions in fibrotic remodeling. The present study focused on SOCS1, a target gene of miR-155 that is elevated and suppresses macrophages during inflammatory responses. Previous studies have reported that SOCS1 activates associated inflammatory molecules as a potent inhibitor of the production and release of cytokines (34-36). It is well established that miR-155 confers competitive fitness to regulatory $\mathrm{T}$ cells by targeting SOCS1 (37). In addition, it was previously confirmed that miR-155 targets SOCS1 to activate the interleukin-6/Janus kinase/signal transducer and activator of transcription 3 signaling pathway for $\mathrm{T}$ helper 17 cell differentiation (38). Furthermore, another study demonstrated that the expression of miR-155 in macrophages was a potent contributor to cardiac hypertrophy and failure in pressure overload conditions, and SOCS1 knockdown restored the hypertrophy-stimulating potency in miR-155 knockout macrophages (18). It has also been reported that overexpression of miR-155 promoted the proliferation and invasion of human laryngeal squamous cell carcinoma by targeting SOCS1 (39). In the current study, the results demonstrated that the expression of SOCS1 was induced in heart tissue and cardiac fibroblasts by Ang II; the expression of SOCS1 was lower in miR-155 KO mice compared with WT mice. The results indicate that the absence of miR-155 may inhibit profibrotic remodeling by targeting SOCS1. TGF- $\beta 1$, the most potent inducer of ECM production, may promote fibroblast to myofibroblast differentiation. The TGF- $\beta 1 /$ SMAD3-mediated signaling pathway is reported to have a pivotal role in ECM metabolism (40). Overexpression of miR-155 in the present study led to reduced levels of SOCS1 and hyperactivation of profibrotic TGF- $\beta 1 /$ SMAD3 signaling, which results in excessive fibrosis and adverse ventricular remodeling. However, a limitation of the present study was that the derepression of SOCS1 in miR-155 KO mice was not prevented. Therefore, deletion of SOCS1 in heart and isolated cardiac fibroblasts should be performed to validate the role of SOCS1 in miR-155-regulated cardiac fibrosis. In future studies, we aim to further investigate the associations between and among miR-155, SOCS1 and TGF- $\beta 1 /$ SMAD3 signaling. In addition, other limitations of the present study exist; the number of the mice in each group is relatively small and, considering that there appears to be inflammatory cell infiltration in the Ang II hearts, it is not easy to determine whether intrinsic cells of the heart (myocytes or fibroblasts) or inflammatory cells are the most important source of cells for modulation of SOCS1 by miR-155. The mechanisms underlying miR-155-induced cardiac fibrotic remodeling therefore require further investigation.

The current study addressed the role of miR-155 and its potential regulatory mechanism in cardiac fibrosis induced by Ang II. Both genetic inactivation and pharmacological inhibition of miR-155 reduced the production of collagen, which indicates that miR-155 may be an important contributor to cardiac fibrosis induced by Ang II. Furthermore, it was demonstrated that miR-155 facilitated fibroblast to myofibroblast transformation in isolated cardiac fibroblasts. Clinically, pharmacological inhibitors targeting miR-155 may become a useful adjunct in the treatment of Ang II-dependent cardiac remodeling. However, the mechanisms that drive cardiac fibrotic remodeling in response to pressure overload require further investigation. Further studies concerning miR-155, particularly human studies, are required. In conclusion, inhibition of miR-155 reduced fibroblast to myofibroblast transformation and ameliorated the progression of cardiac fibrotic remodeling induced by Ang II. Therefore, inhibition of endogenous miR-155 may have clinical potential in alleviating cardiac fibrosis in heart failure.

\section{Acknowledgements}

The present study was supported by a grant from the National Natural Science Foundation of China (grant no. 81170205).

\section{References}

1. Lorenzen JM, Schauerte C, Hübner A, Kölling M, Martino F, Scherf K, Batkai S, Zimmer K, Foinquinos A, Kaucsar T, et al: Osteopontin is indispensible for AP1-mediated angiotensin II-related miR-21 transcription during cardiac fibrosis. Eur Heart J 36: 2184-2196, 2015.

2. González A, Ravassa S, Beaumont J, López B and Díez J: New targets to treat the structural remodeling of the myocardium. J Am Coll Cardiol 58: 1833-1843, 2011.

3. Díez J, Querejeta R, López B, González A, Larman M and Martinez Ubago JL: Losartan-dependent regression of myocardial fibrosis is associated with reduction of left ventricular chamber stiffness in hypertensive patients. Circulation 105: 2512-2517, 2002 .

4. Chen W and Frangogiannis NG: Fibroblasts in post-infarction inflammation and cardiac repair. Biochim Biophys Acta 1833: 945-953, 2013.

5. Melchior-Becker A, Dai G, Ding Z, Schäfer L, Schrader J, Young MF and Fischer JW: Deficiency of biglycan causes cardiac fibroblasts to differentiate into a myofibroblast phenotype. J Biol Chem 286: 17365-17375, 2011.

6. Thum T and Lorenzen JM: Cardiac fibrosis revisited by microRNA therapeutics. Circulation 126: 800-802, 2012.

7. Kvakan H, Luft FC and Muller DN: Role of the immune system in hypertensive target organ damage. Trends Cardiovasc Med 19: 242-246, 2009.

8. Lee RC and Ambros V: An extensive class of small RNAs in Caenorhabditis elegans. Science 294: 862-864, 2001.

9. Small EM and Olson EN: Pervasive roles of microRNAs in cardiovascular biology. Nature 469: 336-342, 2011.

10. Rawal S, Manning P and Katare R: Cardiovascular microRNAs: As modulators and diagnostic biomarkers of diabetic heart disease. Cardiovasc Diabetol 13: 44, 2014.

11. Blahna MT and Hata A: Regulation of miRNA biogenesis as an integrated component of growth factor signaling. Curr Opin Cell Biol 25: 233-240, 2013. 
12. Papageorgiou N, Tsalamandris S, Giolis A and Tousoulis D: MicroRNAs in cardiovascular disease: Perspectives and reality. Cardiol Rev 24: 110-118, 2016.

13. Duisters RF, Tijsen AJ, Schroen B, Leenders JJ, Lentink V, van der Made I, Herias V, van Leeuwen RE, Schellings MW, Barenbrug $\mathrm{P}$, et al: $\mathrm{miR}-133$ and $\mathrm{miR}-30$ regulate connective tissue growth factor: Implications for a role of microRNAs in myocardial matrix remodeling. Circ Res 104: 170-178, 2009.

14. Thum T, Gross C, Fiedler J, Fischer T, Kissler S, Bussen M, Galuppo P, Just S, Rottbauer W, Frantz S, et al: MicroRNA-21 contributes to myocardial disease by stimulating MAP kinase signalling in fibroblasts. Nature 456: 980-984, 2008.

15. Elton TS, Selemon H, Elton SM and Parinandi NL: Regulation of the MIR155 host gene in physiological and pathological processes. Gene 532: 1-12, 2013.

16. Nazari-Jahantigh M, Wei Y, Noels H, Akhtar S, Zhou Z, Koenen RR, Heyll K, Gremse F, Kiessling F, Grommes J, et al: MicroRNA-155 promotes atherosclerosis by repressing Bcl6 in macrophages. J Clin Invest 122: 4190-4202, 2012.

17. Seok HY, Chen J, Kataoka M, Huang ZP, Ding J, Yan J, Hu X and Wang DZ: Loss of MicroRNA-155 protects the heart from pathological cardiac hypertrophy. Circ Res 114: 1585-1595, 2014

18. Heymans S, Corsten MF, Verhesen W, Carai P, van Leeuwen RE, Custers K, Peters T, Hazebroek M, Stöger L, Wijnands E, et al: Macrophage microRNA-155 promotes cardiac hypertrophy and failure. Circulation 128: 1420-1432, 2013.

19. Bala S, Csak T, Saha B, Zatsiorsky J, Kodys K, Catalano D, Satishchandran A and Szabo G: The pro-inflammatory effects of miR-155 promote liver fibrosis and alcohol-induced steatohepatitis. J Hepatol 64: 1378-1387, 2016.

20. Shyu KG, Lu MJ, Chang H, Sun HY, Wang BW and Kuan P: Carvedilol modulates the expression of hypoxia-inducible factor-1alpha and vascular endothelial growth factor in a rat model of volume-overload heart failure. J Card Fail 11: 152-159, 2005.

21. Sreejit P, Kumar S and Verma RS: An improved protocol for primary culture of cardiomyocyte from neonatal mice. In Vitro Cell Dev Biol Anim 44: 45-50, 2008.

22. Tanaka A, Ide T, Fujino T, Onitsuka K, Ikeda M, Takehara T, Hata Y, Ylikallio E, Tyynismaa H, Suomalainen A and Sunagawa K: The overexpression of Twinkle helicase ameliorates the progression of cardiac fibrosis and heart failure in pressure overload model in mice. PLoS One 8: e67642, 2013.

23. Weber M, Kim S, Patterson N, Rooney K and Searles CD MiRNA-155 targets myosin light chain kinase and modulates actin cytoskeleton organization in endothelial cells. Am J Physiol Heart Circ Physiol 306: H1192-H1203, 2014.

24. Livak KJ and Schmittgen TD: Analysis of relative gene expression data using real-time quantitative PCR and the 2(-Delta Delta C(T)) method. Methods 25: 402-408, 2001

25. Shyu KG, Ko WH, Yang WS, Wang BW and Kuan P: Insulin-like growth factor-1 mediates stretch-induced upregulation of myostatin expression in neonatal rat cardiomyocytes. Cardiovasc Res 68: 405-414, 2005.

26. Matsusaka H, Ide T, Matsushima S, Ikeuchi M, Kubota T, Sunagawa K, Kinugawa $S$ and Tsutsui $\mathrm{H}$ : Targeted deletion of matrix metalloproteinase 2 ameliorates myocardial remodeling in mice with chronic pressure overload. Hypertension 47: 711-717, 2006
27. Tili E, Croce CM and Michaille JJ: miR-155: On the crosstalk between inflammation and cancer. Int Rev Immunol 28: 264-284, 2009.

28. Faraoni I, Antonetti FR, Cardone J and Bonmassar E: miR-155 gene: A typical multifunctional microRNA. Biochim Biophys Acta 1792: 497-505, 2009.

29. Corsten MF, Papageorgiou A, Verhesen W, Carai P, Lindow M, Obad S, Summer G, Coort SL, Hazebroek M, van Leeuwen R, et al: MicroRNA profiling identifies microRNA-155 as an adverse mediator of cardiac injury and dysfunction during acute viral myocarditis. Circ Res 111: 415-425, 2012.

30. Roy S and Sen CK: MiRNA in innate immune responses: Novel players in wound inflammation. Physiol Genomics 43: 557-565, 2011.

31. Wu D, Lei H, Wang JY, Zhang CL, Feng H, Fu FY, Li L and Wu LL: CTRP3 attenuates post-infarct cardiac fibrosis by targeting Smad3 activation and inhibiting myofibroblast differentiation. J Mol Med (Berl) 93: 1311-1325, 2015.

32. Pan Z, Sun X, Shan H, Wang N, Wang J, Ren J, Feng S, Xie L, Lu C, Yuan Y, et al: MicroRNA-101 inhibited postinfarct cardiac fibrosis and improved left ventricular compliance via the FBJ osteosarcoma oncogene/transforming growth factor- $\beta 1$ pathway. Circulation 126: 840-850, 2012.

33. Divakaran V and Mann DL: The emerging role of microRNAs in cardiac remodeling and heart failure. Circ Res 103: 1072-1083, 2008.

34. Oury C, Servais L, Bouznad N, Hego A, Nchimi A and Lancellotti P: MicroRNAs in valvular heart diseases: Potential role as markers and actors of valvular and cardiac remodeling. Int J Mol Sci 17: pii: E1120, 2016.

35. Heinrich PC, Behrmann I, Haan S, Hermanns HM, Müller-Newen G and Schaper F: Principles of interleukin (IL)-6-type cytokine signalling and its regulation. Biochem J 374: $1-20,2003$

36. Cittadini A, Monti MG, Iaccarino G, Castiello MC, Baldi A, Bossone E, Longobardi S, Marra AM, Petrillo V, Saldamarco L, et al: SOCS1 gene transfer accelerates the transition to heart failure through the inhibition of the gp130/JAK/STAT pathway. Cardiovasc Res 96: 381-390, 2012.

37. Xu HF, Fang XY, Zhu SH, Xu XH, Zhang ZX, Wang ZF, Zhao ZQ, Ding YJ and Tao LY: Glucocorticoid treatment inhibits intracerebral hemorrhage-induced inflammation by targeting the microRNA-155/SOCS1 signaling pathway. Mol Med Rep 14 3798-3804, 2016.

38. Lu LF, Thai TH, Calado DP, Chaudhry A, Kubo M, Tanaka K, Loeb GB, Lee H, Yoshimura A, Rajewsky K and Rudensky AY: Foxp3-dependent microRNA155 confers competitive fitness to regulatory $\mathrm{T}$ cells by targeting SOCS1 protein. Immunity 30 : 80-91, 2009.

39. Yao R, Ma YL, Liang W, Li HH, Ma ZJ, Yu X and Liao YH: MicroRNA-155 modulates Treg and Th17 cells differentiation and Th17 cell function by targeting SOCS1. PLoS One 7: e46082, 2012.

40. Zhao XD, Zhang W, Liang HJ and Ji WY: Overexpression of miR-155 promotes proliferation and invasion of human laryngeal squamous cell carcinoma via targeting SOCS1 and STAT3. PLoS One 8: e56395, 2013. 